\title{
Response to a large rotavirus outbreak on South Tarawa, Kiribati, 2013
}

\author{
Teanibuaka Tabunga, ${ }^{a}$ Maryanne Utiera, ${ }^{a}$ Rosemary Tekoaua, ${ }^{a}$ Tebikau Tibwe, ${ }^{a}$ Teatao Tira, ${ }^{a}$ Tebuka Toatu, ${ }^{b}$ \\ Sala Elbourne Duituturaga, ${ }^{b}$ Eric Nilles ${ }^{c}$ and Adam Craig ${ }^{c}$ \\ Correspondence to Teanibuaka Tabunga (e-mail: teanibuaka.tebunga@gmail.com)
}

Introduction: In July 2013, during annual independence celebrations in Kiribati, staff at Tungaru Central Hospital on South Tarawa reported an increase in children presenting with severe diarrhoea. This report describes the outbreak investigation, findings and response.

Method: After notification of the outbreak, all health facilities on South Tarawa began reporting cases of acute diarrhoea and/or vomiting through the early warning syndromic surveillance system on a daily basis. Community awareness was raised and the public was encouraged to present to a health facility if ill with acute gastroenteritis. Specimens were collected and sent for laboratory testing.

Results: Between 10 and 24 July 2013, 1118 cases of gastroenteritis were reported; 103 were hospitalized and six died. The median age of cases was one year (range: 0-68 years); 93.4\% were aged less than five years. Rotavirus was identified in $81 \%$ of specimens tested. The outbreak response included enhanced surveillance, community education, clinical training and changes to in-hospital patient management for infection control.

Discussion: This outbreak was the largest diarrhoea outbreak in Kiribati in five years. Factors that may have contributed to the magnitude and severity of the outbreak included high household density, inadequate sanitation infrastructure and a mass gathering - all increasing the chance of transmission - as well as limited clinical response capacity. The current outbreak highlights the importance of clinical management to minimize severe dehydration and death. Rotavirus vaccination should be considered as an adjunct to other comprehensive enteric disease control measures as recommended by the World Health Organization.

$\mathrm{K}$ iribati is located in the Pacific Ocean and consists of one volcanic island and 32 low-lying atolls. Despite being spread over 3.5 million $\mathrm{km}^{2}$ of ocean, the total land area is only $811 \mathrm{~km}^{2}{ }^{1}$ The population of Kiribati in 2010 was 103058 people, with an average population density of 128 per $\mathrm{km}^{2}$. Almost half (48.7\%) of the population live on the capital islands - the islets that make up South Tarawa and the atoll of Betio.

South Tarawa (including Betio) is a string of low lying islets that stretches $23 \mathrm{~km}$ from Betio to Tanaea (Figure 1). South Tarawa is less than $3 \mathrm{~m}$ above the sea level, with an average width of $450 \mathrm{~m}$, has a total of $16 \mathrm{~km}^{2}$, of which $10 \mathrm{~km}^{2}$ is usable. ${ }^{2}$ The population density on South Tarawa is 3184 persons per $\mathrm{km}^{2}$ with a household density of seven to eight people per household, making South Tarawa among the most densely populated areas of the world. ${ }^{2,3}$ Residents of
South Tarawa (and especially the islet of Betio) experience high rates of respiratory infections, diarrhoea and dysentery. High incidence of these illnesses have been linked to overcrowding. ${ }^{1,2}$ Kiribati's routine childhood vaccination programme does not include rotavirus vaccine.

In mid-July each year Kiribati celebrates its independence with a weeklong national holiday. Celebrants visiting South Tarawa result in further overcrowding and increased pressure on water, environmental and food hygiene infrastructures.

On 10 July 2013, mid-way through the 2013 independence celebrations, staff from the Tungaru Central Hospital (TCH) on South Tarawa reported to the Ministry of Health and Medical Services (MHMS) Public Health Division through the established syndromic

\footnotetext{
Ministry of Health and Medical Services, Public Health Division, Kiribati.

Secretariat of the Pacific Community, Public Health Division.

World Health Organization Division of Pacific Technical Support, Emerging Disease Surveillance and Response Unit, Suva, Fiji. Submitted: 22 November 2013; Published: 30 May 2014 doi: $10.5365 /$ wpsar.2013.4.4.006
} 
Figure 1. Map of South Tarawa, Kiribati

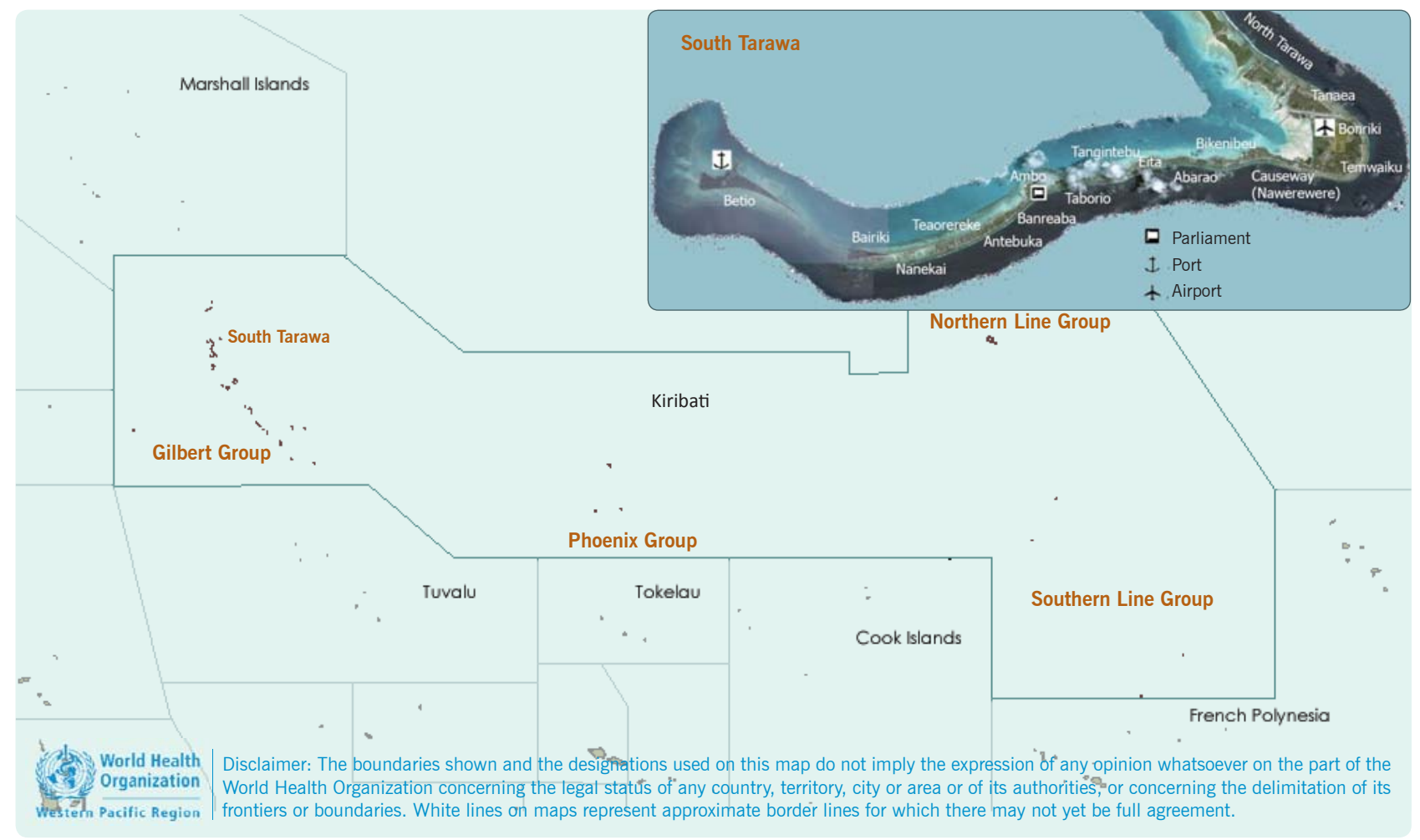

Note: Inset map of South Tarawa, Kiribati was adapted from: http://commons.wikimedia.org/wiki/File:06_Map_of_South_Tarawa,_Kiribati.jpg.

surveillance system that 20 children had presented with severe acute diarrhoea. This potential outbreak was subsequently investigated, and this report describes the outbreak investigation, findings and response.

\section{METHODS}

The Kiribati Syndromic Surveillance System, which is part of the regional Pacific Syndromic Surveillance System, was enhanced for this investigation by adding a specific outbreak case definition. All health facilities (two hospitals and 14 community clinics) on South Tarawa reported cases that met the case definition through the existing reporting mechanisms. The outbreak case definition was: "any person presenting to a health facility on South Tarawa with acute diarrhoea and/ or vomiting after 10 July 2013". Health facility staff applied the outbreak case definition along with their routine syndromic surveillance activities for the duration of the outbreak. Health facilities reported the number of presentations, as well as any unusual events (e.g. particularly severe cases or deaths) to the National Health Information Systems Unit (HIS) for collation, analysis and dissemination of information.
Rotavirus enzyme-linked immunosorbent assay testing was performed at the Fiji Centre for Disease Control Laboratory on suspect-case stool samples. Genotyping of rotavirus-positive samples was conducted at the WHO Collaborating Centre, Murdoch Childrens Research Institute, Melbourne, Australia.

Clinical and public health control measures were implemented at the health facilities and in the community. Control measures included health promotion and enhancement of clinical care capacity.

\section{RESULTS}

\section{Epidemiological investigation}

From 10 to 24 July 2013, 1118 cases met the outbreak case definition on South Tarawa (attack rate: $2.3 \%$ ), of which 103/1118 (9.2\%) required hospitalization and $6 / 1118$ died (case fatality rate: $0.54 \%$ ). Males constituted 566 cases (50.6\%), and the median age was one year (mean: 2.9 years; range: zero to 68 years). Most cases (1044/1118, 93.4\%) and all deaths were less than five years old; the attack rate among this group was $13 \%$ (Table 1 ). 
Table 1. Number of suspected cases and incidence proportion by sex, age and village of residence

\begin{tabular}{|c|c|c|c|c|}
\hline & Population (2010) & $\begin{array}{c}\text { Number of suspected } \\
\text { cases }\end{array}$ & $\begin{array}{c}\text { Proportion of } \\
\text { suspected cases (\%) }\end{array}$ & $\begin{array}{c}\text { Rate } \\
\text { (per } 10000 \text { population) }\end{array}$ \\
\hline \multicolumn{5}{|l|}{ Sex } \\
\hline Male & 24233 & 566 & 50.6 & 233.6 \\
\hline Female & 25949 & 551 & 49.3 & 212.3 \\
\hline Unknown & NA & 1 & 0.1 & ND \\
\hline \multicolumn{5}{|l|}{ Age groups (years) } \\
\hline$<5$ & 8043 & 1044 & 93.4 & 1298.0 \\
\hline $5-14$ & 9076 & 30 & 2.7 & 33.1 \\
\hline $15-49$ & 25222 & 33 & 3.0 & 13.1 \\
\hline $50+$ & 5841 & 9 & 0.8 & 15.4 \\
\hline Unknown & NA & 2 & 0.2 & ND \\
\hline \multicolumn{5}{|l|}{ Village of residence } \\
\hline Abariao & 1665 & 26 & 2.3 & 156.2 \\
\hline Ambo & 2200 & 34 & 3.0 & 154.5 \\
\hline Antebuka & 1087 & 16 & 1.4 & 147.2 \\
\hline Bairiki & 3524 & 80 & 7.2 & 227.0 \\
\hline Banreaaba & 1969 & 21 & 1.9 & 106.7 \\
\hline Betio & 15755 & 548 & 49.0 & 347.8 \\
\hline Bikenibeu & 6568 & 78 & 7.0 & 118.8 \\
\hline Bonriki & 2355 & 19 & 1.7 & 80.7 \\
\hline Causeway (Nawerewere) & 2054 & 26 & 2.3 & 126.6 \\
\hline Eita & 3061 & 86 & 7.7 & 281.0 \\
\hline Nanikai & 988 & 6 & 0.5 & 60.7 \\
\hline Taborio & 1282 & 24 & 2.2 & 187.2 \\
\hline Tanaea & 279 & 3 & 0.3 & 107.5 \\
\hline Tangintebu & 89 & 8 & 0.7 & 898.9 \\
\hline Teaoraereke & 4171 & 48 & 4.3 & 115.1 \\
\hline Temwaiku & 3135 & 41 & 3.7 & 130.8 \\
\hline Unknown & NA & 54 & 4.8 & ND \\
\hline Total & 50182 & 1118 & 100.0 & 222.8 \\
\hline
\end{tabular}

NA - not applicable; ND - not determined.

Note: Some columns may not add up to $100 \%$ due to the rounding off of decimal places.

The first reported case presented to the emergency department of TCH on 10 July 2013. The number of new presentations peaked on 18 July 2013 and returned to pre-outbreak levels by 24 July 2013 (Figure 2). Most cases ( $n=988 ; 88.5 \%$ ) reported suffering acute diarrhoea, and 759 cases (67.9\%) reported acute vomiting. Fever was reported in 21 (1.9\%) cases.

The majority of cases resided in the villages of Betio and Bairiki at the western end of South Tarawa and Tangintebu and Eita on central South Tarawa. The other $45.4 \%$ of cases resided in villages geographically dispersed along South Tarawa (Figure 1; Table 1).

\section{Laboratory investigation}

Of the 20 specimens collected, 16 returned a result with $13(81 \%)$ positive for rotavirus. Eight rotavirus-positive specimens were forwarded for genotyping and were all identified as G3P. Tests for other infectious agents returned negative results.

\section{Control measures}

The clinical response to the outbreak included:

- providing clinical staff with training on appropriate diagnosis and case management; 
Figure 2. Outbreak epidemic curve of the number of suspected cases, by reported date of illness onset, South Tarawa, Kiribati, July 2013

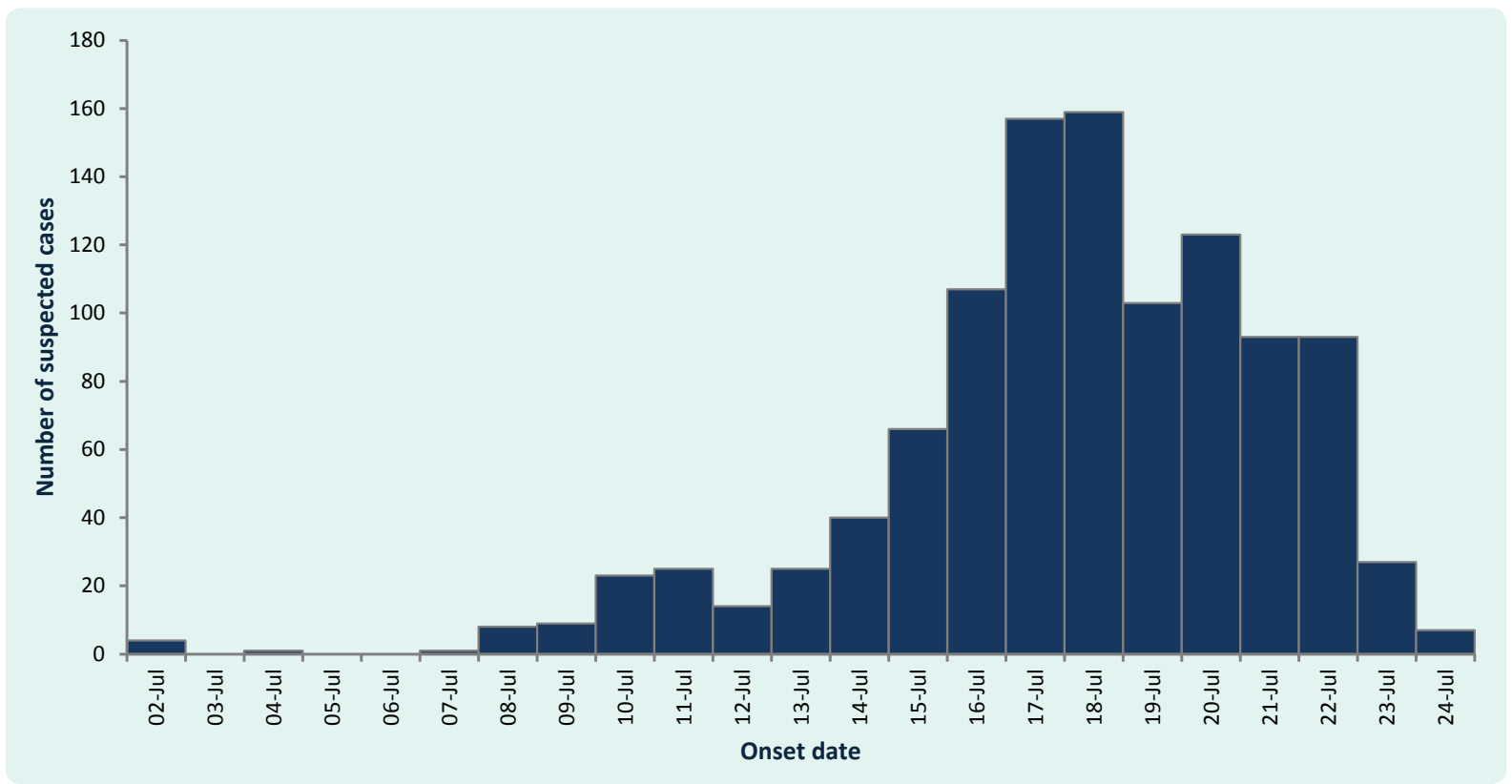

- implementing a modified triage system at hospitals;

- increasing ward space to accommodate admitted patients and to prevent hospital-based transmission; and

- increasing staff numbers at hospitals by transferring clinical staff from community clinics.

Community clinics extended their hours of operation (with four open 24 hours a day) to enhance health care accessibility. Pharmacy supplies were distributed to all health facilities on South Tarawa.

A health promotion campaign for hand hygiene, environmental sanitation and food safety was conducted through local radio village talks, announcements during church services and in locations where risk of transmission is high (i.e. kava bars, child care centres, primary schools). This campaign continued for approximately three weeks after the number of cases had returned to pre-outbreak levels and was extended to reach outlining islands.

Surveillance was enhanced on atolls neighbouring South Tarawa, with atoll health facilities notified and requested to report patients that met the outbreak case definition. A short-lived slight increase in the number of presentations with acute diarrhoea increased in the period immediately after the South Tarawa outbreak was reported.

During the outbreak the MHMS issued situation updates once a day to relevant government and nongovernment stakeholders. The updates also formed the basis of public communication messages released by MHMS.

\section{DISCUSSION}

This outbreak was the largest diarrhoeal outbreak experienced in Kiribati in five years, with 1118 cases and an incidence proportion of $13 \%$. Rotavirus G3P, a common genotype of rotavirus circulating globally, was implicated in the majority of suspected cases tested. Rotavirus is the most common cause of severe diarrhoeal disease among infants and young children globally and is estimated to be responsible for over two million hospitalizations (mainly for severe dehydration) and 527000 deaths annually with $85 \%$ of deaths occurring in low-income countries, usually due to late presentation to hospital or inadequate capacity to provide appropriate clinical care. ${ }^{4}$ Rotavirus is primarily transmitted by the faecal-oral route and may be present in contaminated water. $^{5}$

The outbreak response caused significant strain on both the clinical and public health systems of Kiribati and raised anxiety among the population; however, it 
was considered effective. The outbreak demonstrated the importance of having strong management structures for public health events, including a designated event commander to oversee response activities and preparedness plans. Having pre-approved and protocoldriven surveillance and response arrangements in place before the outbreak occurred made the response easier to manage. Such arrangements must be easily activated, understood by all involved and adaptable to account for ever-changing situations. The MHMS has invested much effort to develop the national health system's preparedness for public health emergencies. These capacities have been developed to help meet Kiribati's obligations to the International Health Regulations (2005). ${ }^{6}$ Reference laboratory testing was facilitated by the laboratory network of the Pacific Public Health Surveillance Network. ${ }^{7}$

Communication between the event commander and relevant response managers was critical for coordination. The early detection of this outbreak highlighted the role played by early warning syndromic surveillance in Kiribati. Further, the reach of the Kiribati Syndromic Surveillance System (all health facilities on South Tarawa) and the ability to use the system's wellestablished reporting mechanisms meant that enhanced surveillance was implemented quickly across all sites and data were reported to the HIS in a streamlined manner. This greatly enhanced the speed at which data were shared and lessened the burden of data capture and management placed upon staff at the national level. It is noted that as the outbreak case definition was based on presentations to a health facility, the number of cases identified is likely to be less than the true number of people affected.

Mortality from childhood diarrhoea is overwhelmingly secondary to severe dehydration. This outbreak highlights the importance of a systematic and rapid assessment for dehydration followed by either oral or intravenous rehydration, or resuscitation based upon findings. Most cases of childhood diarrhoea can be managed with zinc and low-osmolality oral rehydration solution (ORS), but a small proportion of severely dehydrated children - or children with persistent vomiting - will require intravenous rehydration or occasionally urgent intravenous resuscitation. Breast milk is an excellent rehydration fluid and should be encouraged, together with ORS, for children still breastfeeding. In addition to fluid replacement, children with diarrhoea should continue to be fed during their illness as food intake supports fluid absorption, and helps maintain nutritional status and the body's ability to fight infection. Zinc treatment may be used to help reduce the duration and severity of diarrhoea and hence fluid loss. ${ }^{5,8}$ Severely ill children requiring hospitalization should remain under medical supervision until recovered or risk of relapse has passed.

This outbreak highlights the importance of rotavirus as an epidemic pathogen and a potentially important role for rotavirus vaccination as one element in a comprehensive programme to control causes of diarrhoeal disease. In 2009, WHO recommended that rotavirus vaccine be included in all national immunization programmes and considered a priority, particularly in countries with high rotavirus gastroenteric-associated mortality rates; ${ }^{8}$ to date, rotavirus vaccine has not been implemented in Kiribati. Given the high rate of infection in low-income countries and reported high level of protection offered by the rotavirus vaccination, consideration of vaccine use is recommended. Rotavirus vaccination is reported to offer $40-90 \%$ protection against rotavirus gastroenteritis after one and/or two years of follow up. ${ }^{8}$ WHO provides guidelines for the implementation of population-wide rotavirus vaccination.

This outbreak coincided with a mass gathering on South Tarawa, an event that likely affected the spread and severity of the outbreak. The gathering likely changed the outbreak transmission dynamics by increasing the density of the population on the atolls and increasing the chance of poorly handled, cooked or stored food. Health facility staff vacations for the gathering reduced response capacity. In the future, risk assessment to identify and determine the impact mass gatherings may have on health and health systems capacity as well as pre-emptive public health planning is advised.

\section{Conflicts of interest}

None declared.

Funding

None. 


\section{Acknowledgements}

We acknowledge the Fiji Centre for Disease Control Laboratories (Mataika House) and the WHO Collaborating Centre at Murdoch Childrens Research Institute in Melbourne for their support with the testing and genotyping of specimen samples. We also acknowledge the work of clinical and public health staff of MHMS.

\section{References}

1. Western Pacific Country Health Information Profiles. Manila, World Health Organization Regional Office for the Western Pacific, 2011.

2. Republic of the Kiribati Island Report Series: 6 South Tarawa. South Tarawa, Kiribati, 2012.
3. Report on the Kiribati 2010 Census of Population and Housing. South Tarawa, Kiribati National Statistics Office, 2012.

4. 'Rotavirus'. Geneva, World Health Organization, 2013 (http://www. who.int/nuvi/rotavirus/en/, accessed 10 October 2013).

5. Haymann DL. Control of Communicable Diseases Manual, 19th Edition. Washington, DC, American Public Health Association and World Health Organization, 2008.

6. Craig A, Kool J, Nilles E. The Pacific experience: supporting small island countries and territories to meet their 2012 International Health Regulation (2005) commitments. Western Pacific Surveillance and Response, 2013, 4:1-5. doi:10.5365/ wpsar.2012.3.4.007

7. Pacific Public Health Surveillance Network. World Health Organization, Secretariat of the Pacific Community and Fiji National University, 2013 (http://www.spc.int/phs/pphsn/index. htm, accessed 5 November 2013).

8. World Health Organization. Rotavirus vaccine: WHO position paper - January 2013. Weekly epidemiological Report No 5, 2013, 49-64. 